\title{
Acute myeloid leukemia with an unusual histologic pattern mimicking metastatic carcinoma in bone marrow: a diagnostic pitfall
}

\author{
Mingyi Chen • Young Kim • Qin Huang • \\ Karen Chang $\cdot$ Karl K. Gaal $\cdot$ Lawrence M. Weiss
}

Received: 7 March 2011 / Accepted: 28 April 2011 /Published online: 17 May 2011

(C) Springer-Verlag 2011

\begin{abstract}
We describe two cases of acute myeloid leukemia with an unusual histological pattern mimicking metastatic non-hematopoietic neoplasm, leading to diagnostic difficulties. Both patients presented with pancytopenia and absence of circulating blasts. Bone marrow aspirations were not successful. Bone marrow core biopsies contained neoplastic cells arranged in cohesive sheets or single file patterns with prominent myxoid stroma resembling metastatic carcinoma. The accompanying bone marrow touch imprints showed clusters of immature cells with monocytoid features. The cell lineage was further revealed by immunohistochemical analysis of bone marrow core biopsy sections. The neoplastic cells showed strong reactivity for myeloperoxidase, CD33, CD43, $\mathrm{CD} 45$, and $\mathrm{CD} 68$, indicating a myeloid cell origin with monocytic differentiation. One patient (age, 39 years) received standard chemotherapy for acute myeloid leukemia, which resulted in remission of bone marrow disease. The other patient (age, 85 years) died with multiple organ failure before treatment. Failure to recognize these unusual histological features of acute myeloid leukemia could result in misdiagnosis from bone marrow biopsy.
\end{abstract}

Keywords Hematologic malignancy Acute myeloid leukemia $\cdot$ Metastatic carcinoma

M. Chen · Y. Kim • Q. Huang • K. Chang • K. K. Gaal •

L. M. Weiss

Department of Pathology, City of Hope National Medical Center,

1500 East Duarte Road,

Duarte, CA 91010, USA

M. Chen $(\varangle)$

Department of Pathology and Laboratory Medicine,

University of California Davis Medical Center,

PATH Bldg. 4400V Street,

Sacramento, CA 95817, USA

e-mail: mingyi.chen@ucdmc.ucdavis.edu

\section{Introduction}

Acute myeloid leukemias (AML) with monocytic differentiation often present with marked leukocytosis containing many circulating blasts and leukemic infiltrates in lymph nodes, liver, spleen, and soft tissues [1, 2]. In the peripheral blood, the number of monocytic cells is usually more than $5.0 \times 10^{9} / \mathrm{L}$. However, on rare occasions, there may be a low peripheral white blood cell count with the absence of circulating blasts. The diagnosis of acute myeloid leukemia can still be made by bone marrow biopsy with the aid of immunohistochemical and cytogenetic studies.

AML involving a packed hypercellular bone marrow can exhibit a variety of histological patterns. At rare times, AML can present histologically with carcinomatoid features and almost all cases reported to date have involved extramedullary sites [3, 4]. In addition, AML can mimic metastatic carcinoma in the skin, lymph nodes, and solid organs with neoplastic cells in cohesive sheets, thus rendering a diagnosis of granulocytic sarcoma [5]. However, similar-appearing examples of acute leukemia in the bone marrow itself are only rarely described [6]. In this context, we report two cases of AML with monocytic differentiation showing a unique histological pattern that resembles metastatic carcinoma in the bone marrow.

\section{Report of two cases}

Case 1

An 85-year-old woman was admitted to a regional hospital, complaining of fever, weakness, and leg pain. Her past medical history included colectomy without chemotherapy for colon cancer 10 years prior. No lymphadenopathy or 
organomegaly was detected by physical examination and CT scan. Hemoglobin concentration was $9.4 \mathrm{~g} / \mathrm{dL}$, white cell count $3.9 \times 10^{9} / \mathrm{L}$, and platelet count $59.0 \times 10^{9} / \mathrm{L}$. The peripheral blood smear differential count was reported as follows: $18.0 \%$ neutrophils, $14.0 \%$ bands, $26.0 \%$ lymphocytes, $18.0 \%$ monocytes, $13.0 \%$ metamyelocytes, $8.0 \%$ myelocytes, $2.0 \%$, promyelocytes, myelocytes, $1.0 \%$ eosinophils, $0.0 \%$ basophils, and $0.0 \%$ blasts. Dysplastic granulocytes were not seen. Bone marrow aspiration yielded a dry tap, thus causing difficulty in interpretation of the bone marrow touch imprint and core biopsy. The case was sent to our institution for consultation where a diagnosis of acute myelomonocytic leukemia was established by immunohistochemical studies. The patient died within a week of being diagnosed and before treatment could be initiated. An autopsy was not performed.

\section{Case 2}

A 39-year-old man with an unremarkable past medical history was investigated for pancytopenia. Computed tomographic examination of the abdomen showed a moderately enlarged spleen and a slightly enlarged liver. Hemoglobin concentration was $6.9 \mathrm{~g} / \mathrm{L}$; hematocrit, $20.3 \%$; MCV, $89.4 \mathrm{fl}$; white blood cell count, $1.9 \times 10^{9} / \mathrm{L}$; and platelet count, $9.0 \times 10^{9} / \mathrm{L}$. The peripheral blood smear differential count was reported as follows: $33.0 \%$ lymphocytes, $59.0 \%$ neutrophils and bands, $4.0 \%$ monocytes, $4.0 \%$ eosinophils, $0.0 \%$ basophils, and $0.0 \%$ blasts. The peripheral blood smear showed no circulating blasts, monocytosis, or dysplastic neutrophils. Multiple bone marrow aspirations yielded either dry taps or paucicellular smears. The hemodiluted marrow aspirate smears showed scattered immature monocytes. The possibility of marrow involvement by a histiocytic tumor was considered by the referring pathologist. Difficulty in interpretation of the bone marrow aspirate and biopsy material led to referral consultation at our institution where the diagnosis of acute monocytic leukemia was established. The patient was successfully treated with standard chemotherapy for AML and remission was achieved.

\section{Materials and methods}

Bone marrow aspirate smears and biopsy sections were prepared using standard methods. Aspirate smears were stained with Wright-Giemsa and biopsy sections were stained with hematoxylin-eosin. Immunohistochemical studies were performed using formalin-fixed, paraffinembedded tissue sections using a heat-induced epitope retrieval technique and a universal secondary antibody kit that used a peroxidase-conjugated labeled dextran poly- mer (EnVision + System, Peroxidase; DAKO, Carpinteria, CA, USA) [7, 8]. The specific antibodies include lysozyme $(1: 2,000)$, myeloperoxidase (1:500), tryptase (1:200), S-100 (1:3,000), Villin (1:500), CDX-2 (1:200), CD1a (1:100), CD3 (1:150), CD20 (1:500), CD23 (1:500), CD30 (1:120), CD31 (1:300), CD33 (1:100), CD43 (1:300), CD45 (1:300), CD61 (1:200), CD68 (1:500), CD117 (1:50), CD123 (1:200), and CD163 (1:500) (DAKO, Carpinteria, CA, USA), CD1a (1:2, Beckman Coulter Inc, Fullerton, CA, USA), CD34 (1:100, BD Biosciences, San Jose, CA, USA), CD56 (1:100, Zymed, Carlsbad, CA, USA), Langerin glycoprotein $(1: 100$, Novocastra Laboratories Ltd, Newcastle upon Tyne, UK), Pax-5 (1:100, BD Biosciences, San Diego, CA, USA), Cytokeratin (Oscar, 1:100, Zeta Corporation, Arcadia, CA, USA), and CAM 5.2 (1:50, BectonDickinson, Mountain View, CA, USA). Appropriate positive and negative tissue control samples were used throughout the tests.

The methods for cytogenetic and fluorescence in situ hybridization (FISH) studies were the same as previously described [9]. Cytogenetic analysis was performed on bone marrow aspirates using standard techniques. GTG banding identified the individual chromosomes. A minimum of 20 metaphase cells per sample was analyzed. Triple-color FISH analysis was performed on bone marrow touch imprints or paraffin embedded bone trephine biopsy sections by using specific probes to determine the number of interphase nuclei containing the fusion gene. In general, 200 interphase nuclei were analyzed per case. Screening for the NPM1 mutations was carried out with a LightCycler-based melting curve assay by polymerase chain reaction (PCR) analysis as previously described $[10,11]$.

\section{Results}

\section{Case 1}

The H\&E stained bone marrow core biopsy sections showed a hypercellular marrow with nodular and interstitial infiltrates by sheets of large atypical cells. The tumor cells showed trabecular or cord-like patterns of growth with prominent myxoid stroma (Fig. 1). A moderate increase in marrow reticulin fibrosis was noted. On high magnification, the malignant cells showed markedly pleomorphic nuclei with irregular contours, fine chromatin, occasional nucleoli, and moderate amounts of pale basophilic cytoplasm with numerous cytoplasmic vacuoles. Numerous atypical mitotic figures were observed. The residual trilineal hematopoietic elements showed a spectrum of maturation without dysplastic features. Reticulin stain revealed an increase of reticulin 

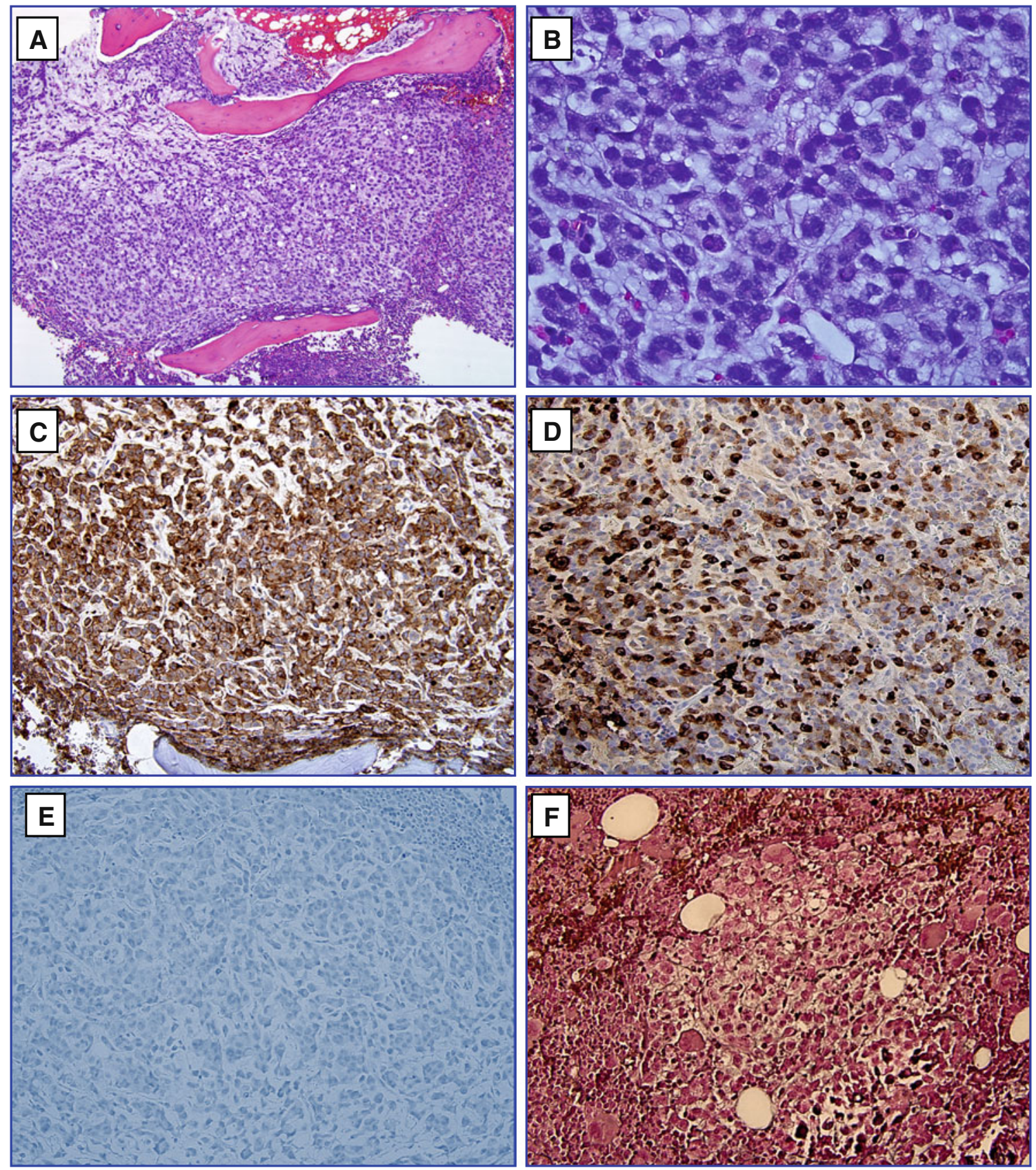

Fig. 1 a Hypercellular bone marrow (case 1) with sheets of large atypical cells infiltrating in trabecular/cord patterns with prominent myxoid stroma mimicking the appearance of "metastatic carcinoma". b On higher magnification, the single-file cords of immature cells show marked pleomorphic nuclei with irregular contours, hyperchromatic or dispersed chromatin, and prominent nucleoli. By

fibers in loose networks (MF-1). No overt collagen fibrosis was detected by trichrome stain.

Immunohistochemical studies The immature cells reacted with CD33, CD43, CD45, CD56 (focal), CD68, CD117 (focal), and myeloperoxidase (focal). No reactions were seen with cytokeratins (OSCAR and CAM5.2), Villin, CDX-2,

immunohistochemistry, the neoplastic cells are positive for CD33 (c), and myeloperoxidase (d), but are negative for Cytokeratin (OSCAR) (e). Reticulin stain highlights the marrow fibrosis (MF-1) (f). Hematoxylin-eosin, original magnifications, $\times 100$ (a) and $\times 600$ (b). Immunohistochemistry with hematoxylin counterstain, original magnification $\times 200(\mathbf{c}-\mathbf{f})$

lysozyme, CD1a, CD3, CD20, CD23, CD30, CD31, CD34, CD61, CD123, CD163, PAX-5, Langerin, S100, and tryptase. Overall, the combined morphological and immunohistochemical features of the blasts established the diagnosis of acute myelomonocytic leukemia. The primary diagnosis of metastatic carcinoma of colonic origin was excluded by the negative epithelial cell markers. 
The conventional cytogenetic study showed 46, XY [20], normal male karyotype. The FISH study on core biopsy sections for 11q23 (MLL locus) was negative. No insertion mutations at exon 12 of the NPM1 gene were detected by PCR.

\section{Case 2}

The hemodiluted bone marrow aspirate smears were paucicellular containing a few scattered large, immature cells with an increased nuclear to cytoplasmic ratio, fine chromatin, variable nucleoli, and scant to moderate amounts of basophilic cytoplasm with a few fine azurophilic granules (Fig. 2). The rare residual granulocytic and erythroid precursors showed normal maturation without overt dysplastic features. No atypical lymphoid population was seen. Megakaryocytes appeared decreased in number with normal morphology. A 200 cell differential count on the aspirate smears revealed: $76.0 \%$ immatureappearing atypical cells, $5.0 \%$ promonocytes, $1.0 \%$ myelocytes/metamyelocytes, $5.0 \%$ bands/segmented neutrophils, $1.0 \%$ eosinophils, $2.0 \%$ lymphocytes, and $10.0 \%$ erythroid precursors. The trephine and clot biopsy sections revealed an interstitial infiltrate of immature appearing large atypical cells occurring singly or in clusters with abundant myxoid cytoplasm. The residual trilineal hematopoietic elements were markedly decreased. Reticulin stain revealed an increase of reticulin fibers in loose networks (MF-1). No overt collagen fibrosis was detected by Trichrome stain.

Immunohistochemcal studies The monocytic lineage for the immature-appearing atypical cells was confirmed as follows: The immature cells were positive for CD33, CD43, CD45, and CD68, and were negative for other histiocytic/dendritic cell related markers including CD1a, CD21, CD163, Langerin, and S-100. The combined morphological and immunohistochemical features of the blasts established a diagnosis of acute monocytic leukemia. The referring pathologist's consideration of marrow involvement by a metastatic histiocytic tumor was ruled out.

The conventional cytogenetic study was unsuccessful due to the low cell yield. The FISH study on touch imprints for 11q23 (MLL locus) was negative. No insertion mutations at exon 12 of the NPM1 gene were detected by PCR.

\section{Discussion}

We present two cases of AML with monocytic differentiation that histologically resembles metastatic carcinoma. Due to the inadequacy of bone marrow aspirate smears, the diagnosis greatly depended on the bone marrow biopsies. Immunohistochemistry and other ancillary studies were imperative in excluding metastatic malignancy or a rare lymphoid disorder.

Carcinomas or sarcomas can metastasize to the bone marrow and often manifest at high clinical stages. Therefore, the evaluation of bone marrow involvement by metastasis is crucial [12]. Identifying the primary site of origin for a metastases is dependent on the morphologic findings, immunohistochemical profile, and most importantly, clinical history. Usually, epithelial tumors (carcinomas) tend to form groups of cohesive cells with a desmoplastic stromal reaction that are typically easy to

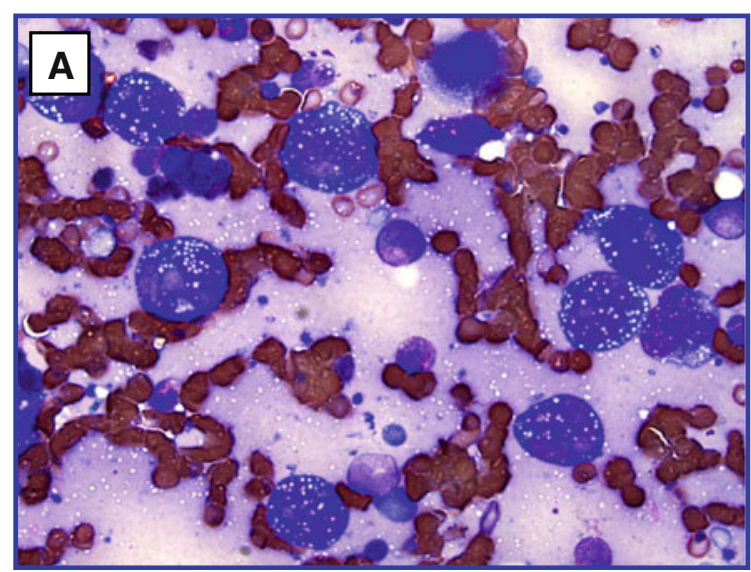

Fig. 2 a Bone marrow aspirate (case 2) with scattered large atypical immature cells. b Hypercellular bone marrow with a prominent infiltrate of large atypical cells. The residual trilineage hematopoiesis is markedly decreased. The neoplastic cells have large irregular nuclei,

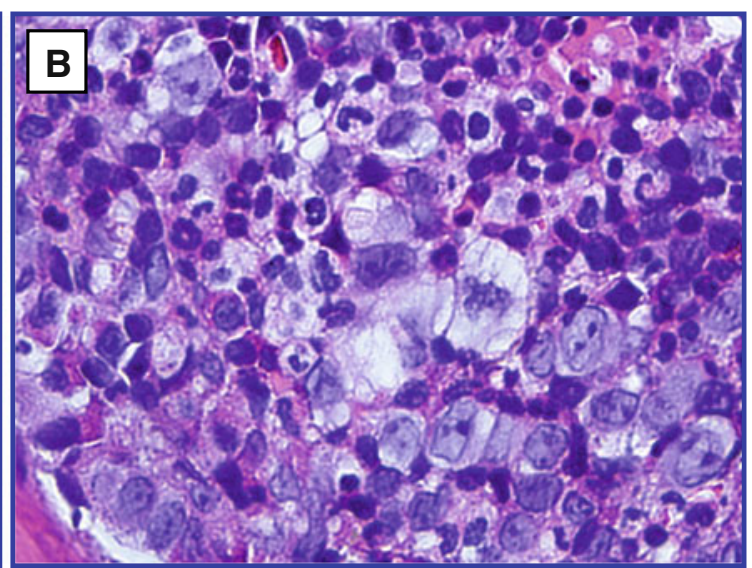

dispersed chromatin, variable nucleoli, and abundant pale basophilic cytoplasm with many cytoplasmic vacuoles. Wright-Giemsa stain, original magnification, $\times 600$ (a); hematoxylin-eosin, original magnifications, $\times 400$ (b) 
distinguish from normal hematopoietic cells. On rare occasions, myeloid neoplasia can exhibit some unusual histologic patterns with cellular features mimicking a carcinoma.

When the diagnosis of acute myeloid neoplasm is considered, the differential diagnosis includes acute megakaryoblastic leukemia [13], acute panmyelosis with myelofibrosis [13], MDS/AML associated with myelofibrosis [14] and rarely cytokeratin-positive acute myeloid leukemia [15]. Therefore, a complete panel of immunophenotypical, cytogenetic, and molecular studies is necessary in order to render an accurate final diagnosis $[8,9]$. The expression of monocytic markers such as CD14, CD4, CD11b, CD11c, CD64, CD68, CD36, and lysozyme in the blasts supports the diagnosis of acute myeloid leukemia with monocytic differentiation [2]. The megakaryoblasts can be recognized by positive reactions to von Willbrand's factor, CD41, CD61, and CD42b [13]. In the literature, very few cases of acute myelomonocytic leukemia cases have been reported to be aberrantly positive for cytokeratins by the "cocktail" antibodies (AE1/AE3 and CAM 5.2), therefore resembling metastatic carcinoma [15]. However, the positive staining for myeloid markers and negative staining for other epithelial markers in the blasts can be helpful in establishing the correct diagnosis [7].

In acute leukemia, the malignant leukemic stem cells can accumulate multiple mutations and evolve into new cellular clones $[1,16]$. Monocytic neoplasms histologically mimicking carcinoma have been reported $[3,6]$. It has been suggested that the tendency to appear cohesive may be an inherent characteristic of neoplastic cells with monocytic differentiation. The literature has commented on the role of the adhesion molecules or homing receptors of the leukemia cells in the formation of this variant pattern [17]. In addition, alterations in the bone marrow microenvironment as a result of the neoplastic process may be involved, rendering a cohesive carcinomatoid appearance [16]. Ancillary studies including immunohistochemistry and cytogenetics are needed for an accurate diagnosis [18].

A neoplastic process associated with marked marrow fibrosis can result in failed marrow aspiration (dry tap) as illustrated in case 1 . The diagnosis of acute leukemia can be very challenging in patients who present with pancytopenia without circulating blasts or accompanying dysplasia. We have described two cases in which the blasts of acute myelomonocytic or monocytic leukemia appeared cohesive, resembling metastatic carcinoma involving the bone marrow. This unusual pattern can present a diagnostic dilemma. In both instances, these features led to an initial provisional diagnosis of metastatic neoplasm in the bone marrow, and therefore conventional cytochemical stains for leukemias, such as myeloperoxidase or nonspe- cific esterase, were omitted. Awareness of these rare histological patterns in AML and the application of appropriate immunohistochemical studies can prevent delayed or missed diagnosis.

In summary, our cases represent examples of AML with monocytic differentiation in the bone marrow showing histologic features that mimick metastatic carcinoma. These cases call attention to the complexity of AML with unusual histological features.

Acknowledgments The authors are grateful to Dr. Ralph Green for critical reading of the manuscript.

Conflict of interest The authors declare we have no conflict of interest.

\section{References}

1. Arber DA, Brunning RD, Orazi A, Porwit A (2008) Acute myeloid leukemia, not otherwise specified, 4th edn. IARC Press, Lyon, pp 130-134

2. Vardiman JW et al (2009) The 2008 revision of the World Health Organization (WHO) classification of myeloid neoplasms and acute leukemia: rationale and important changes. Blood 114:937951

3. McIlwain L, Sokol L, Moscinski LC, Saba HI (2003) Acute myeloid leukemia mimicking primary testicular neoplasm. Presentation of a case with review of literature. Eur J Haematol 70:242-245

4. Menasce LP, Banerjee SS, Beckett E, Harris M (1999) Extramedullary myeloid tumour (granulocytic sarcoma) is often misdiagnosed: a study of 26 cases. Histopathology 34:391398

5. Pileri SA et al (2007) Myeloid sarcoma: clinico-pathologic, phenotypic and cytogenetic analysis of 92 adult patients. Leukemia 21:340-350

6. Hamoudi WH, Medeiros LJ, Townsend G, Glassman AB, BuesoRamos CE (2000) Acute myelomonocytic leukemia with histologic features resembling sarcomatoid carcinoma in bone marrow. Arch Pathol Lab Med 124:315-318

7. Chu PG, Weiss LM (2002) Keratin expression in human tissues and neoplasms. Histopathology 40:403-439

8. Chu PG, Chang KL, Arber DA, Weiss LM (1999) Practical applications of immunohistochemistry in hematolymphoid neoplasms. Ann Diagn Pathol 3:104-133

9. Arber DA, Stein AS, Carter NH, Ikle D, Forman SJ, Slovak ML (2003) Prognostic impact of acute myeloid leukemia classification. Importance of detection of recurring cytogenetic abnormalities and multilineage dysplasia on survival. Am J Clin Pathol 119:672-680

10. Huang Q, Chen W, Gaal KK, Slovak ML, Stein A, Weiss LM (2008) A rapid, one step assay for simultaneous detection of FLT3/ITD and NPM1 mutations in AML with normal cytogenetics. Br J Haematol 142:489-492

11. Pasqualucci L et al (2006) Mutated nucleophosmin detects clonal multilineage involvement in acute myeloid leukemia: impact on WHO classification. Blood 108:4146-4155

12. Krishnan C, George TI, Arber DA (2007) Bone marrow metastases: a survey of nonhematologic metastases with immunohistochemical study of metastatic carcinomas. Appl Immunohistochem Mol Morphol 15:1-7 
13. Orazi A et al (2005) Acute panmyelosis with myelofibrosis: an entity distinct from acute megakaryoblastic leukemia. Mod Pathol 18:603-614

14. Weinberg OK et al (2009) Clinical characterization of acute myeloid leukemia with myelodysplasia-related changes as defined by the 2008 WHO classification system. Blood 113:1906-1908

15. Turner JJ, Milliken S (2000) A case of keratin-positive acute myeloid leukemia: a possible role for cytokeratin 19 as a specific epithelial marker. Pathology 32:98-101
16. Dick JE, Lapidot T (2005) Biology of normal and acute myeloid leukemia stem cells. Int J Hematol 82:389-396

17. Liesveld JL (1997) Expression and function of adhesion receptors in acute myelogenous leukemia: parallels with normal erythroid and myeloid progenitors. Acta Haematol 97:53-62

18. Arber DA, Carter NH, Ikle D, Slovak ML (2003) Value of combined morphologic, cytochemical, and immunophenotypic features in predicting recurrent cytogenetic abnormalities in acute myeloid leukemia. Hum Pathol 34:479-483 